\title{
Evolution of magnetic fields in galaxies and future observational tests with the Square Kilometre Array
}

\author{
T. G. Arshakian ${ }^{1}$, R. Beck ${ }^{1}$, Marita Krause ${ }^{1}$, and D. Sokoloff ${ }^{2}$
}

\author{
1 Max-Planck-Institut für Radioastronomie, Bonn, Germany \\ e-mail: [tarshakian;rbeck;mkrause] @mpifr-bonn.mpg.de \\ 2 Department of Physics, Moscow State University, Russia \\ e-mail: sokoloff@dds.srcc.msu.su
}

Received 14 September 2008 / Accepted 16 October 2008

\begin{abstract}
Aims. In the context of models of galaxy formation and evolution, we investigate the cosmological evolution of large- and small-scale magnetic fields inside galaxies.

Methods. We use the dynamo theory to derive the timescales of amplification and ordering of magnetic fields in disk and puffy galaxies. Turbulence in protogalactic halos generated by thermal virialization can drive an efficient turbulent dynamo. Results from simulations of hierarchical structure formation cosmology provide a tool to develop an evolutionary model of regular magnetic fields coupled with galaxy formation and evolution.

Results. The turbulent (small-scale) dynamo was able to amplify a weak seed magnetic field in halos of protogalaxies to a few $\mu \mathrm{G}$ strength within a few $10^{8} \mathrm{yr}$. This turbulent field served as a seed to the mean-field (large-scale) dynamo. Galaxies similar to the Milky Way formed their disks at $z \approx 10$ and regular fields of $\mu \mathrm{G}$ strength and a few kpc coherence length were generated within 2 Gyr (at $z \approx 3$ ), but field-ordering on the coherence scale of the galaxy size required an additional $6 \mathrm{Gyr}($ at $z \approx 0.5)$. Giant galaxies formed their disks at $z \approx 10$, allowing more efficient dynamo generation of strong regular fields (with kpc coherence length) already at $z \approx 4$. However, the age of the Universe is short for fully coherent fields in giant galaxies larger than $15 \mathrm{kpc}$ to have been achieved. Dwarf galaxies should have hosted fully coherent fields at $z \approx 1$. After a major merger, the strength of the turbulent field is enhanced by a factor of a few.

Conclusions. This evolutionary scenario can be tested by measurements of polarized synchrotron emission and Faraday rotation with the planned Square Kilometre Array (SKA). We predict an anticorrelation between galaxy size and ratio between ordering scale and galaxy size. Weak regular fields (small Faraday rotation) in galaxies at $z \lessgtr 3$ are signatures of major mergers. Undisturbed dwarf galaxies should host fully coherent fields, giving rise to strong Faraday rotation signals. Radio observations may serve as a clock for measuring the time since the last major merger.
\end{abstract}

Key words. galaxies: formation - galaxies: evolution - galaxies: magnetic fields - galaxies: high-redshift - galaxies: interactions radio continuum: galaxies

\section{Introduction}

Polarized synchrotron emission and Faraday rotation inferred the presence of regular large-scale magnetic fields with spiral patterns in the disks of nearby spiral galaxies (Beck 2005), which were successfully reproduced by mean-field dynamo theory (Beck et al. 1996; Rüdiger \& Hollerbach 2004; Shukurov 2005). It is therefore natural to apply dynamo theory also in predicting the generation of magnetic fields in young galaxies at high redshifts. However, there is a lack of observational information with which to test models. The available data (Kronberg et al. 2008; Bernet et al. 2008; Wolfe et al. 2008; see also the review by Kronberg 1994), although limited, imply that the environments of galaxies are significantly magnetized at high redshifts $(z \lesssim 3)$, with regular magnetic-field strengths comparable to or higher than those at the current epoch. Another indication of strong magnetic fields in young galaxies is the tight radio far-infrared correlation, which is valid in galaxies at least to distances of $z \simeq 3$ (Seymour et al. 2008). To explain the almost linear correlation, the total magnetic-field strength must be related to the star-formation rate (Lisenfeld et al. 1996; Niklas $\&$ Beck 1997). Since observations at high redshifts are biased towards starburst galaxies, strong magnetic fields presumably do exist in distant objects.

We now have sufficient evidence that strong magnetic fields were present in the early Universe and that synchrotron emission should be detected with future radio telescopes such as the Square Kilometre Array (SKA). The SKA will spectacularly increase the sensitivity and angular resolution of radio observations and allow us to observe an enormous number of distant galaxies at similar resolution to that achievable for nearby galaxies today (van der Hulst et al. 2004).

The dynamo was introduced in to astrophysics as a mechanism for transforming the kinetic energy of the motions of electrically conductive media into magnetic energy. This concept describes the amplification of small-scale as well as largescale, regular fields. For practical, mathematical reasons, the dynamo equation was separated into a small-scale and a large-scale part, two aspects of the same mechanism with widely different timescales, but often considered as two independent dynamo models.

The amplification of large-scale magnetic fields and the general growth of magnetic energy are not the only merits of galactic dynamos. For the evolution of galactic magnetic fields, the 
transformation of a turbulent into a regular field is of similar value. It is the large-scale regular field whose observation is most spectacular and whose generation has attracted most attention in astrophysics. As far as we know today, the generation of regular magnetic fields is a delicate process that requires large-scale rotation of the body under consideration. This rotation produces the $\alpha$-effect, which amplifies the magnetic field, and differential rotation ensures that this amplification is effective. This defines the classical "mean-field" galactic dynamo as discussed below, taking into account recent results from numerical simulations of galaxy formation.

Developing a consistent dynamo model to describe the entire lifetime of a galaxy requires information about the basic physical parameters controlling dynamo action and their evolution. Regular large-scale magnetic fields can be generated and amplified by the mean-field galactic dynamo in high-redshift galaxies, provided that a gaseous, rotating disk already exists. The formation of disk galaxies and the epoch of this formation are fundamental problems in astronomy. High resolution numerical simulations of disk formation in galaxies demonstrated that a dynamical disk could be formed at redshifts $z \sim 5-6$ and even higher (Governato et al. 2007; Mayer et al. 2008). A more robust understanding of the history of magnetism in young galaxies may help to solve fundamental cosmological questions about the formation and evolution of galaxies (Gaensler et al. 2004).

In the absence of large-scale rotation, turbulent motions can amplify magnetic fields, which are, in general, irregular, smallscale fields. This process is therefore known as the turbulent or small-scale dynamo (e.g. Zeldovich et al. 1990). The growth in magnetic fluctuations is clearly visible in many numerical MHD simulations of the interstellar medium (Meneguzzi et al. 1981; Hanasz et al. 2004; de Avillez \& Breitschwerdt 2005; Kowal et al. 2006; Iskakov et al. 2007; Gressel et al. 2008; Wang \& Abel 2008). We note that all numerical simulations of the generation of regular magnetic fields in disk galaxies begin at the disk formation epoch; we assume that seed fields of strength between a few $\mu \mathrm{G}$ and $10^{-3} \mu \mathrm{G}$ exists already in the disk and do not consider the field amplification up to this level.

High-resolution simulations of protogalactic clouds demonstrated that significant turbulence can be generated prior to disk formation during the thermal virialization of the halo (Wise \& Abel 2007). Strong turbulence at these early epochs may drive a turbulent dynamo, which can effectively amplify the halo magnetic fields. The amplified magnetic fields can form the basis of a seed field for the generation of regular fields in the disk of a newly formed galaxy.

The first model of dynamo action in young galaxies by Beck et al. (1994) proposed a two-stage dynamo, a turbulent dynamo to amplify a weak, seed field in a disk within less than a Gyr and a large-scale dynamo to amplify the regular field within a few Gyr. However, some of the assumptions, e.g. the epoch of disk formation, the large disk thickness and the constant radius, are in conflict with the modern scenario of galaxy formation.

Dynamo theory has been well developed as a branch of MHD and, as a first step, we exploit mainly conventional approaches to galactic dynamos (see e.g. Ruzmaikin et al. 1988; Beck et al. 1996; Rüdiger \& Hollerbach 2004) rather than more recent findings in dynamo theory (see e.g. Tobias \& Cattaneo 2008). In this paper, we develop a simplified model for the evolution of magnetic fields in both protogalactic halos and galaxies based on the recent numerical developments in the study of the formation and evolution of galaxies during the epoch of hierarchical structure formation. We use the known timescales of the turbulent dynamo and mean-field dynamo in puffy and disk galaxies to explore the earliest generation and evolution of regular magnetic fields in halos and to describe the main phases of magnetic-field evolution in dwarf and disk galaxies, as well as the influence of merging events on the magnetic evolution. In Sect. 2, we derive the timescales of field amplification and field ordering in disk and spherical galaxies by the mean-field dynamo. The timescale of the turbulent dynamo is discussed in Sect. 3, and the origin of seed magnetic fields in Sect. 4. In Sect. 5, we summarize developments in the formation and evolution of disk and dwarf galaxies, and in Sect. 6, we develop an evolutionary model of regular magnetic fields in galaxies. Perspectives on detecting polarized radio emission from nearby and from distant galaxies are discussed in Sect. 7, and conclusions are drawn in Sect. 8.

Throughout the paper, a flat cosmology model $\left(\Omega_{\Lambda}+\Omega_{\mathrm{m}}=1\right)$ is used with $\Omega_{\mathrm{m}}=0.3$ and $H_{0}=70 \mathrm{~km} \mathrm{~s}^{-1} \mathrm{Mpc}^{-1}$.

\section{Magnetic-field generation in galaxies by the mean-field dynamo}

In this Section, we refer to thin-disk galaxies that rotate differentially. Theoretical analysis of differentially rotating disks demonstrated that a disk is only locally stable to axisymmetric perturbations if the Toomre's stability criterion is fulfilled (Julian \& Toomre 1966), i.e. only cool disks in which both the gas and stars have low velocity dispersion can survive for a long time (Binney \& Tremaine 1987). Hence, the velocity dispersion in disk galaxies at higher redshifts is probably similar to that in nearby galaxies. In the following, we adopt the usual values of $v=10 \mathrm{~km} \mathrm{~s}^{-1}$ and a scale of turbulence of $l=100 \mathrm{pc}$. Turbulence in galaxy disks is assumed to be driven by supernovae (Ruzmaikin et al. 1988; Korpi et al. 1999; de Avillez \& Breitschwerdt 2005; Gressel et al. 2008). The other basic parameters of the galactic mean-field dynamo are the angular velocity of the galactic rotation $\Omega$, the disk thickness $h$, the disk radius $R$, and the gas density $\rho$.

\subsection{Timescales of regular magnetic fields in disk galaxies}

The conventional theory of the galactic mean-field dynamo in disk galaxies allows the generation of a regular galactic magnetic field as a result of joint action of differential rotation $\Omega$ and helical turbulent motions of interstellar gas. The latter is responsible for the so-called $\alpha$-effect $(\alpha)$. According to Ruzmaikin et al. (1988), the intensity of both generators can be characterized by two dimensionless numbers, $R_{\omega}$ and $R_{\alpha}$ :

$$
R_{\omega}=\frac{1}{\beta}\left(\frac{h}{500 \mathrm{pc}}\right)^{2}\left(\frac{\Omega}{20 \mathrm{~km} \mathrm{~s}^{-1} \mathrm{kpc}^{-1}}\right)
$$

and

$R_{\alpha}=\frac{\alpha}{\beta}\left(\frac{h}{500 \mathrm{pc}}\right)$

where $\Omega$ and $h$ are the angular velocity of the galaxy and the half-thickness of a galactic disk (normalized to the galactic angular velocity and half-thickness, respectively) and $\beta$ is the turbulent diffusivity. A flat rotation curve $(r \partial \Omega / \partial r \sim \Omega)$ was assumed in deriving the above relations. The parameters $\alpha$ and $\beta$ cannot be measured directly from observations, and our aim is to reduce them to observable parameters. According to simple mixing length theory the turbulent diffusivity is

$\beta=\frac{l v}{3}$ 
where $l$ is the basic length scale of turbulence, $v$ is the turbulent velocity, and the numerical factor characterizes the dimensions of the space. Typical numbers, as found from observations of nearby galaxies, are $l=100 \mathrm{pc}$ and $v=10 \mathrm{~km} \mathrm{~s}^{-1}$ (e.g. Ruzmaikin et al. 1988). The $\alpha$-effect is determined by the Coriolis force and density gradient (Krause \& Rädler 1983):

$\alpha=\frac{\Omega l^{2}}{h}$.

The joint action of both generators can be described by a so-called dynamo number:

$\left|D_{\mathrm{d}}\right|=R_{\omega} R_{\alpha} \simeq 9\left(\frac{h \Omega}{v}\right)^{2}$.

The absolute value is taken because $D_{\mathrm{d}}$ is usually considered to be negative. The dynamo growth rate $(\Gamma)$ is given by

$\Gamma=\left|D_{\mathrm{d}}\right|^{1 / 2} \frac{\beta}{h^{2}}$,

where $\beta / h^{2}$ is the time of turbulent diffusion. The dynamo timescale for amplification of the regular field is $t^{*}=\Gamma^{-1}$ ( $e$-folding time), and we derive

$t^{*}=\frac{h}{\Omega l}$

from Eqs. (3), (5) and (6). This estimate is the result of combining standard estimates from galactic dynamo theory, which has not been presented before.

During its lifetime $T$, a galaxy completes $N$ rotations. Assuming $\Omega \approx 20 \mathrm{~km} \mathrm{~s}^{-1} \mathrm{kpc}^{-1}, T=10^{10}$ years and $h / l \approx 5$, we derive $N \approx 30$ and $t^{*} \approx 5 T /(2 \pi N) \approx 2.5 \times 10^{8} \mathrm{yr}$. Hence, the conventional galactic mean-field dynamo can amplify the regular magnetic field by a factor of at most $10^{17}$, while in practice it can be lower (see Sect. 4).

The galactic dynamo is a threshold phenomenon. The regular magnetic field is only maintained (or amplified) if the dynamo number is larger than a critical value $\left|D_{\mathrm{d}}\right|>\left|D_{\mathrm{cr}, \mathrm{d}}\right|$. Ruzmaikin et al. (1988) derived $\left|D_{\mathrm{cr}, \mathrm{d}}\right| \simeq 7$ from the numerical simulations of galactic dynamo models, which they determined to be the minimal value required for the dynamo effect to overcome turbulent diffusion. If $\left|D_{\mathrm{d}}\right|<\left|D_{\mathrm{cr}, \mathrm{d}}\right|$, the regular magnetic-field decays. The typical value of dynamo number for the Milky Way (called MW hereafter) is $\left|D_{\mathrm{d}}\right| \simeq 9$ (note that $h \Omega / v \approx 1$ for the MW), indicating that the dynamo mechanism acts in the MW. If $h \Omega / v \ll 1$, a disk galaxy cannot amplify or maintain regular magnetic fields by means of the mean-field dynamo mechanism.

The expression for $t^{*}$ (Eq. (7)) can be written in terms of the magnetic-field strength. The time required to amplify the largescale magnetic field from $B_{0}$ to $B_{*}$ is given by

$\hat{t}=t_{*} \ln \left(B_{*} / B_{0}\right) ;$

If $\hat{t} \geq T$, the dynamo is in its kinematic regime until the present time. Hence, the minimum seed field required for dynamo action is

$B_{0}=B_{*} \exp \left(-T / t^{*}\right)$.

We note that $t^{*}$ is the e-folding timescale for amplification of the magnetic field. The resulting magnetic field is spatially ordered on a coherence scale $l_{\mathrm{c}}$ of a few kiloparsecs $(\mathrm{kpc})$, depending on the disk half-thickness. Full ordering of the field on the coherence scale of the radius $R\left(l_{\mathrm{c}}=R\right)$ of a galaxy takes a far longer time. According to Moss et al. (1998), this ordering timescale $\tilde{t}$ is

$\tilde{t}=\frac{l_{\mathrm{c}}}{\sqrt{\Gamma \beta}}=\frac{h^{2}}{\beta} \frac{l_{\mathrm{c}}}{h}\left|D_{\mathrm{d}}\right|^{-1 / 4}$.

Magnetic-field ordering on the scale of $l_{\mathrm{c}}$ is not simply an instability that can be adequately described by an $e$-folding time but a more delicate process called "front propagation" (the so-called Kolmogorov-Petrovsky-Piskunov effect, Kolmogorov et al. 1937, for review in the context of dynamo theory, see also Zeldovich et al. 1990). For a crude estimate, one can use $\tilde{t}$ from Eq. (10) in the sense that, for times $t<\tilde{t}$, the ordering remains in progress, while for $t>\tilde{t}$, the ordering process is already finalized.

Equation (10) can be presented in terms of diffusion timescales in the vertical $\left(T_{h}\right)$ and radial $\left(T_{\mathrm{r}}\right)$ directions. The timescale for full ordering $\tilde{t}\left(l_{\mathrm{c}}=R\right)$ is far longer than the amplification timescale $t^{*}$ (see factor $R / h$ in Eq. (10)) as well as the diffusion timescale in the vertical direction:

$T_{h}=\frac{h^{2}}{\beta} \simeq 710^{8} \mathrm{yr}$

and $\tilde{t} \ll T_{r}$,

$T_{r}=\frac{R^{2}}{\beta} \simeq 400 T_{h} \gg T$.

The ordering timescale is governed by the geometrical mean of both timescales

$\tilde{t}\left(l_{\mathrm{c}}=R\right)=\sqrt{T_{h} T_{R}}\left|D_{\mathrm{d}}\right|^{-1 / 4} \approx \frac{R}{l}\left(\frac{h}{v \Omega}\right)^{\frac{1}{2}}$.

For MW-type galaxies, $\tilde{t}$ is only a few times shorter than the galaxy lifetime $T$, indicating that transient field configurations, substantially different from the leading eigenmode of the galactic dynamo, can survive in some galaxies until now. This may explain e.g. the large-scale field reversals observed in the MW.

After the ordering timescale $\tilde{t}$, a steady-state magnetic field is maintained. The configuration of this field is expected to be similar to the leading eigenmode of the galactic dynamo, saturated by nonlinear effects (see Sect. 2.2).

\subsection{Equilibrium magnetic-field strength in disk galaxies}

The initial growth of the regular magnetic field due to the galactic dynamo is saturated at some level, which is known as the equilibrium magnetic-field strength $\left(B_{\text {eq }}\right)$ at which dynamo action reaches equilibrium with turbulent dissipation. The equilibrium magnetic-field strength is related to the equipartition field strength $\left(B_{*}\right)$ :

$\frac{\rho v^{2}}{2}=\frac{B_{*}^{2}}{8 \pi}$

or

$B_{*}=v \sqrt{4 \pi \rho}$.

The more elaborated estimate by Shukurov (2004) takes into account the intensity of dynamo action measured by $D_{\mathrm{d}}$ :

$B_{\mathrm{eq}}=B_{*} \sqrt{\frac{D_{\mathrm{d}}}{D_{\mathrm{cr}, \mathrm{d}}}-1}$ 
Using Eq. (5) and $D_{\text {cr }}=7$, we obtain

$B_{\mathrm{eq}}=v \sqrt{4 \pi \rho} \sqrt{1.28\left(\frac{h \Omega}{v}\right)^{2}-1}$.

The concept that $B_{\mathrm{eq}}$ is determined by $B_{*}$ is an oversimplification. A deeper understanding of dynamo saturation based on magnetic helicity conservation (e.g. Kleeorin et al. 2002) yields a similar estimate for $B_{\text {eq }}$.

We conclude that the timescale of dynamo growth and equilibrium field strength are determined by different governing parameters. In particular, if $v$ becomes larger (and the other parameters are fixed), the dynamo timescale remains constant (Eq. (7)), while the equilibrium strength increases. On the other hand, the dynamo number strongly decreases with increasing $v$ (Eq. (5)) and may drop below the critical value.

\subsection{Timescale of regular magnetic fields in quasi-spherical galaxies}

We estimate the dynamo timescales in spherical or "puffy" galaxies with $h / R>0.1$. For a spheroid, $h \approx R$ :

$\left|D_{\mathrm{s}}\right|=R_{\alpha} R_{\omega} \simeq 9\left(\frac{R \Omega}{v}\right)^{2}$,

while the dynamo growth rate is

$\Gamma=\left|D_{\mathrm{s}}\right|^{2 / 3} \frac{\beta}{R^{2}}$

(Sokoloff 2002). This yields the following estimate of the dynamo timescale $t^{* *}$ of a spheroid:

$t^{* *}=\frac{3}{9^{2 / 3}}\left(\frac{v}{R \Omega}\right)^{1 / 3} \frac{R}{\Omega l}$.

We note that the final term is the dynamo timescale for a disk $t^{*}$, where $h$ is replaced by $R$. Assuming that $v=10 \mathrm{~km} \mathrm{~s}^{-1}$ and $R \Omega=250 \mathrm{~km} \mathrm{~s}^{-1}$, we estimate that

$t^{* *}=0.23 t^{*}(R)$,

where $t^{*}(R)$ means that in Eq. (7) $h$ is replaced by $R$. Since $R$ is generally a few times larger than $h$, the timescales for disk and spherical galaxies are more or less comparable.

The dynamo number of a quasi-spherical galaxy is calculated from the ratio of Eq. (7) to Eq. (18), $\left|D_{\mathrm{s}}\right|=\left|D_{\mathrm{d}}\right|(R / h)^{2}=10^{3}-$ $10^{4}$, for $R / h=10$ and $\left|D_{\mathrm{d}}\right|=10-100$ (e.g. Belvedére et al. 1998). The critical dynamo number $\left|D_{\mathrm{cr}, \mathrm{s}}\right|$ for a quasi-spherical body is within the wide range between 300 and $5 \times 10^{3}$ (cf. Sokoloff \& Shukurov 1990 and Sokoloff et al. 2008), depending on the details of the rotation curve and the spatial distribution of the dynamo-governing parameters. For the above estimates, $\left|D_{\mathrm{s}}\right| \approx$ 5000. In this paper, we assume that $\left|D_{\mathrm{s}}\right|>\left|D_{\mathrm{cr}, \mathrm{s}}\right|$ and do not specify the suppression of the mean-field dynamo in spherical bodies.

\section{Magnetic-field generation by the turbulent dynamo}

Magnetic-field generation by the turbulent (small-scale) dynamo in galaxies requires neither large-scale rotation nor a disk, only turbulence. It produces magnetic fields on scales comparable to the basic scale of galactic turbulence on timescales far shorter than that for the conventional mean-field galactic dynamo. The timescale ( $e$-folding time) of the small-scale dynamo is given by

$\tau=l / v$,

(see Batchelor 1950; Landau \& Lifshitz 1959; Kazantsev 1967), while the equilibrium strength $b$ remains comparable to $B_{*}$.

Considering that interstellar turbulence produces random small-scale magnetic fields as well as regular large-scale ones, we compare their strengths. We note that the term $\sqrt{D / D_{\mathrm{cr}}-1}$ in Eq. (16) is usually less than unity, so that the ratio of small-scale and large-scale field strengths $b / B_{\text {eq }}$ is expected to be slightly larger than unity. A ratio of $b / B_{\mathrm{eq}} \simeq 1.7$ is estimated from observations (Ruzmaikin et al. 1988, see however Beck et al. 2003, for a systematic bias imposed on $b / B_{\mathrm{eq}}$ ). For the MW, this ratio, derived from the above theoretical arguments, is

$\frac{b}{B_{\mathrm{eq}}}=\frac{1}{\sqrt{1.28\left(\frac{h \Omega}{v}\right)^{2}-1}} \approx 1.9$,

in good agreement with the observational value.

\section{Origin of seed magnetic fields}

The small-scale and large-scale dynamo mechanisms cannot explain the origin of magnetic fields. Seed magnetic fields are required for the dynamo mechanism to amplify and maintain the magnetic field themselves. One possible origin of a seed field is the Biermann battery mechanism, which was initially suggested in a cosmological context by Harrison (1970) and applied to protogalaxies by Mishustin \& Ruzmaikin (1971) (see Ruzmaikin et al. 1988, for a review). The typical magnetic-field strength produced by the battery mechanism is estimated to be between $B_{\text {seed }}=10^{-20}$ and $10^{-22} \mathrm{G}$.

In the context of a hierarchical structure formation scenario, the Biermann battery is able to generate a field of order $10^{-18} \mathrm{G}$ in a protogalactic cloud at $z \simeq 40$ (Pudritz \& Silk 1989; Davies \& Widrow 2000). This theoretical prediction is consistent with magnetohydrodynamical simulations of Population III star formation by $\mathrm{Xu}$ et al. (2008). Magnetic fields of order $\simeq 10^{-18} \mathrm{G}$ are generated predominantly by the Biermann effect, early in the evolution of the halo $(z=40)$ at low densities and large spatial scales (400 pc).

Other possible mechanisms for generating magnetic seed fields are kinetic plasma instabilities, the so-called Weibel instability (Weibel 1959) in cosmological shocks (Schlickeiser \& Shukla 2003; Medvedev et al. 2004) or cosmological perturbations (proposed by Takahashi et al. 2006) that may produce field strengths of $\sim 10^{-19} \mathrm{G}$ (see also the review by Semikoz \& Sokoloff 2005).

In summary, magnetic fields can be generated by various mechanisms and during various stages of cosmological evolution. It is, however, difficult to generate a regular magnetic field on galactic scales that is of reasonable strength in the pre-recombination era when the Universe was homogeneous and isotropic; the creation of magnetic fields instead requires timescales and peculiar motions with respect to the homogeneous and isotropic cosmological background that are inconsistent with the assumptions of isotropy and homogeneity.

The variety of possible mechanisms for generating seed magnetic fields increases with the development of spatial structures in the Universe. The first magnetic fields that represented seeds of galactic magnetic fields were probably created sometime between the epoch of reionization and formation of galaxies $(10 \lesssim z \lesssim 40)$. 
A mechanism creating small-scale magnetic fields with a vanishing mean value is able to randomly generate a weak largescale field component of

$B_{\mathrm{s}}=b N^{-1 / 2}$

where $N$ is the number of turbulent cells in a galaxy. This is sufficient to represent a seed for the large-scale galactic dynamo, as pointed out by Ruzmaikin et al. (1988), Beck et al. (1996), and Subramanian (1998). The key point is that the number of turbulent cells is large but not enormous, much less then the Avogadro number $A=6 \times 10^{23}$, the typical number of particles in laboratory statistical physics, where terms of order $N^{-1 / 2}$ can be neglected.

Using $N=\pi R^{2} 2 h /\left(4 \pi l^{3} / 3\right) \approx 8 \times 10^{4}$ for a disk galaxy with $R=10 \mathrm{kpc}, h=500 \mathrm{pc}$ and $l=100 \mathrm{pc}$ and assuming that the small-scale magnetic field is on the equipartition level, $b \approx B_{*}$, we obtain

$B_{\mathrm{s}} \approx B_{*} / 300$.

Hence, the large-scale dynamo has to amplify the seed field by only a factor of about 300, much less than the upper limit of the amplification factor of $10^{17}$ obtained from Eq. (7), and the corresponding amplification timescale is then far shorter than the galaxy age.

The evolution of galactic magnetic fields initiated with a seed field generated by the Biermann battery (in the epoch of protogalaxy formation of protogalaxies), which was followed by field amplification by the turbulent small-scale dynamo, and further amplification and ordering by the large-scale dynamo. A deeper understanding of the process would also require inclusion of the magneto-rotational instability (Kitchatinov \& Rüdiger 2004), although this is beyond the scope of the present paper.

\section{Formation and evolution of disk galaxies}

We review results of recent simulations of the hierarchical merging of dark halos, and the formation of protogalaxies and their evolution (no concise review of the recent numerical results exists so far). We attempt to identify the mechanisms responsible for generating and amplifying turbulent and regular magnetic fields in the halo and disk of galaxies, in developing a simple model for the evolution of magnetic fields and determining the earliest epochs at which large-scale regular magnetic fields are formed and amplified in disk galaxies, of the type considered in the next Section. The formation and evolution of regular largescale magnetic fields is related intimately to the formation and evolution of disks in galaxies in terms of geometrical and physical parameters such as the radius $R$, half-thickness $h$, and angular momentum $\Omega$ of the galaxy, turbulence velocity $v$, turbulence scale $l$, and density $\rho$ of the gas. These parameters evolve differently for different galaxy types and sizes.

We adopt the standard hierarchical cold dark matter cosmology in which the cosmic structures and galaxies assemble by the merging of small dark matter halos (Kauffmann et al. 1993; Baugh et al. 1996). The first very massive stars $\left(\gtrsim 100 M_{\odot}\right)$ are predicted to have formed in dark matter halos of virial mass $\sim 10^{6} M_{\odot}$ at redshifts $z \lesssim 20$ (e.g. Tegmark et al. 1997; Omukai $\&$ Palla 2003). The dark matter halos merged, assembling the first protogalaxies of masses $\gtrsim 5 \times 10^{7} M_{\odot}$ at $z \gtrsim 10$
(Press \& Schechter 1974; Rees \& Ostriker 1977). The gas and stellar components of protogalaxies may have had a quasispherical or disk-like distribution, depending on the initial conditions of simulations (Brook et al. 2004; Wise \& Abel 2007; Greif et al. 2008). The first galaxies were able to retain photo-heated gas and maintain self-regulated star formation (e.g. Ricotti et al. 2008), and developed into disk galaxies.

In this scenario, we identify two main cosmological epochs at which magnetic fields can be generated and amplified by different dynamo mechanisms. In the first epoch of the virilization and merging of dark matter halos, which occurred between $35 \gtrsim z \gtrsim 10$, the mass of protogalaxies assembled via gas accretion onto the halos and minor/major mergers of dark matter halos. High-resolution simulations of early pre-galactic halos (Wise \& Abel 2007; Greif et al. 2008), which include primordial gas cooling and mass accretion history, showed that significant turbulence was generated in all cosmological halos during thermal virilization. The gas accreted from the IGM was shockheated to the virial temperature. This mode of accretion (hot accretion) worked effectively at high redshifts in low-mass halos and generated turbulence on scales comparable with the size of the infalling gas. As radiative cooling became efficient, the gas attempted to virialize by increasing its kinetic (turbulent) energy, which was achieved by radial infall and turbulent motions. The turbulence became supersonic with Mach numbers ranging from 1 to 3 (Wise \& Abel 2007).

In this mode (cold accretion), massive filaments could form molecules, which allowed efficient cooling of the filaments and their flow at high velocities $\left(\sim 20 \mathrm{~km} \mathrm{~s}^{-1}\right)$ towards the center of the protogalaxy (Greif et al. 2008). Inflows of cold gas were supersonic with Mach numbers $\sim 10$ and reached the central part of the galaxy, generating significant turbulence with Mach numbers ranging from 1 to 5 .

Virial turbulence may have been most important in halos with efficient radiative cooling rates, for example in halos of masses below $10^{12} M_{\odot}$ that could be cooled effectively by Ly $\alpha$ emission (Birnboim \& Dekel 2003; Dekel \& Birnboim 2006). For low-mass protogalaxies, the largest driving scale of turbulence was $\sim 200 \mathrm{pc}(1 / 3$ of the virial radius) and the rms velocities was around $20 \mathrm{~km} \mathrm{~s}^{-1}$ (John Wise, private communication). The dominant role of virial turbulence played an important role in star formation in the regions with density enhancements and the amplification of magnetic fields by means of the turbulent dynamo (see Sect. 6.1).

Mergers could also have generated turbulence that started on length scales of merging halos and developed to small scales by means of turbulence cascades. Another driver of turbulence was the Kelvin-Helmholtz instability between bulk flows and virialized multi-phase gas (Takizawa 2005), which may have occurred during minor mergers. Simulations of protogalaxies in the redshift range between 35 and 15 demonstrated that the turbulent Mach number depends on merger history. The Mach number was supersonic, reaching values between 2 to 4 in the case of two subsequent major mergers. Before the central collapse occurred in these halos, turbulence was mostly subsonic $(<1$; Wise et al. 2007). Major mergers of halos resulted in high turbulence Mach numbers and hence high turbulent velocities.

The beginning of the second epoch manifested itself by the formation of an extended large-scale disk in the first galaxies. Highly resolved simulations showed that the first galaxies were born at redshifts $z \simeq 10$, after the atomic cooling criterion was fulfilled and they were able to retain the photo-heated gas (Greif et al. 2008). Brook et al. (2004) demonstrated that the thick stellar disk was created during an epoch of multiple mergers of 
gas-rich halos, which were abundant at high redshifts. The population of stars forming during the merging period formed the thick disk. The angular momentum of a significant fraction of the accreted gas (i.e. the angular momentum of the protogalactic halo) resulted in the rotation and flattening of the formed galaxy. The forming thick gas disk was dynamically hot, resulting in the formation of high-velocity stars in the thick disk. After the epoch of violent merger events, the gas was accreted and formed a smooth thin disk.

Recent models of disk formation have included hierarchical dark matter cosmology, gas cooling, star formation, and supernova (SN) feedback (Governato et al. 2007; Stringer \& Benson 2007) and benefitted from the increased resolution of numerical simulations (Mayer et al. 2008). High mass resolution and spatial resolution are important in simulating large MW-type disks (Mayer et al. 2008). The formation of disks at high redshifts $(z>2)$ can be simulated with relatively high spatial and mass resolutions (Governato et al. 2007). The disk started forming in galaxies with $M \gtrsim 10^{11} M_{\odot}$ immediately after the last gas-rich major merger with substantial stellar feedback (Elmegreen et al. 2005; Springel \& Hernquist 2005) at $z_{11 \mathrm{~m}} \sim 2$. Preserving the angular momentum from the merger, the gas cooled and formed the large-scale exponential disk. This effect was more apparent in numerical runs with stronger feedback and higher resolution. After the stellar disk had formed, it remained fairly stable against merger events provided there was sufficient gas to form stars (Springel \& Hernquist 2005). As discussed by Kaufmann et al. (2007), internal and external UV background radiation would prevent cooling below $\sim 10^{4} \mathrm{~K}$ in the warm galactic gas by providing pressure support dynamically comparable to angular momentum support. They showed that pressure support was less important in large halos $\left(\gtrsim 10^{9} M_{\odot}\right)$. This created a tendency to form a thin disk in massive, isolated galaxies, while their less massive counterparts (dwarf galaxies) remained spheroidal, puffy systems with more gas and less efficient star formation than in the larger MW-type galaxies. The disk could settle down (and survive until the present time) in massive halos after the epoch of gas-rich mergers, even at redshifts $z \gtrsim 5$ (Lucio Mayer, private communication). Future higher-resolution numerical simulations will allow to trace the disk formation back to $z \sim 10$.

The advanced disk-formation model presented by Stringer \& Benson (2007) showed that the resulting disks of the isolated galaxy (no merger) and the galaxy formed through minor mergers had very similar evolutionary behaviors. The disk component was not destroyed and evolved into a disk galaxy. The morphology of the formed disk galaxies at $z \lesssim 1.5$ remained unchanged in the case of no merger or only minor mergers, while in the case of frequent minor mergers or a major merger the disk galaxy could evolve into a disk galaxy having a spheroidal or bulge component, or into an elliptical galaxy without disk (see Sect. 6.4).

If a late major merger destroyed the stellar disk at later epochs $(z \sim 1)$, the gaseous and stellar disks could recover, if there was sufficient gas remaining to form new stars.

It is generally believed that, in the hierarchical cold dark matter Universe, the massive disk galaxies were formed first, while the majority of present-day galaxies along the Hubble sequence such as elliptical galaxies and disk galaxies with bulges formed at later epochs by the merging of giant disk galaxies (Kauffmann et al. 1993; Baugh et al. 1996). The existence of massive galaxies at high redshifts is verified by the detections of disk-like or clumpy disk galaxies between $1.4<z<3$ from deep nearinfrared imaging (Labbé et al. 2003) and at $z \sim 5.5$ (Elmegreen et al. 2007). Observations of luminous star-forming galaxies at high angular resolution showed that large and massive rotating protodisk galaxies were present already at $z \sim 2$ to 3 (Genzel et al. 2006).

\section{Evolution of magnetic fields in galaxies}

\subsection{Three-phase model}

In the hierarchical formation scenario, we identify three main phases of magnetic-field evolution in galaxies. In the first phase, the seed magnetic fields of order $\simeq 10^{-18}$ G (Pudritz \& Silk 1989; Davies \& Widrow 2000; Xu et al. 2008) were generated in dark matter halos by the Biermann battery mechanism, well before the formation of first massive stars at $z \sim 20$.

The second phase started at $z \sim 20$ (or $t \sim 0.5 \mathrm{Gyr}$ ) when the merging of halos and virialization generated turbulence in the halo. According to Wise \& Abel (2007), this epoch was dominated by turbulence generated during the thermal virialization of halos. There the turbulent dynamo could effectively amplify the seed magnetic field of halos to the equilibrium level (Eq. (23)), $B_{\mathrm{s}} \sim 20 \mu \mathrm{G}$, on timescales given by Eq. (22).

In the third phase, the mean-field dynamo mechanism started acting in the newly formed galaxies at $z \sim 10$. The first gasrich massive galaxies $\left(\gtrsim 10^{9} M_{\odot}\right)$ formed extended thin disks $(h / R \lesssim 0.1)$ at $z_{\text {disk }}=10$ after major merger events, as discussed in Sect. 5. A weak, large-scale magnetic-field component was generated in the disk from small-scale magnetic fields of the halo (Eq. (24)), amplified to the equilibrium level in the second phase. Then, the "disk" mean-field dynamo amplified and ordered weak, regular magnetic fields on timescales given by Eqs. (7) and (10), respectively. If the formed disk was thick $(h / R \gtrsim 0.1)$ or the disk had not formed, the "quasi-spherical" mean-field dynamo acting in puffy objects would be switched on. It would amplify the field to the equilibrium level and order the magnetic fields on scales of a few kiloparsecs and on timescales given by Eqs. (20) and $(10)(h \approx R)$, respectively. If a thin disk in puffy objects had been formed at later epochs, the "disk" mean-field dynamo would have dominated and amplified the regular fields at the equilibrium level. This scenario is more appropriate for dwarf galaxies $\left(\leqslant 10^{9} M_{\odot}\right)$ that could not maintain the disk at early epochs because of strong UV background radiation, and may have formed a disk at later epochs $(z \sim 2)$ when UV radiation was weak and allowed efficient radiative cooling.

If the disk was destroyed by a late gas-rich, major merger event, the regular magnetic field must have dissipated and increased the amplitude of the turbulent magnetic field. During the disk recovery, the regular magnetic field must have been regenerated and amplified back to its equilibrium level (Eq. (17)). The seed field for the large-scale dynamo is taken from Eq. (24) using $N$ according to the appropriate galaxy radius.

The sketch of the evolution in the amplitude and ordering scale (Eq. (10)) of magnetic fields in dwarf and spiral galaxies is presented in Figs. 1-3.

\subsection{Assumptions of the model}

We assumed an average gas density of a halo (adiabatic model) within its virial radius of about $600 \mathrm{pc}$ at $z=15$ of $\approx 6 \times$ $10^{-24} \mathrm{~g} \mathrm{~cm}^{-3}$ (Wise \& Abel 2007). The gas densities of nearby disk galaxies are calculated to be in the range of between $10^{-24} \mathrm{~g} \mathrm{~cm}^{-3}$ and $15 \times 10^{-24} \mathrm{~g} \mathrm{~cm}^{-3}$ for a random magneticfield strength in the range $\sim(2-10) \mu \mathrm{G}$ (Beck et al. 1996). For simplicity, we assume the same gas density for halo- and 


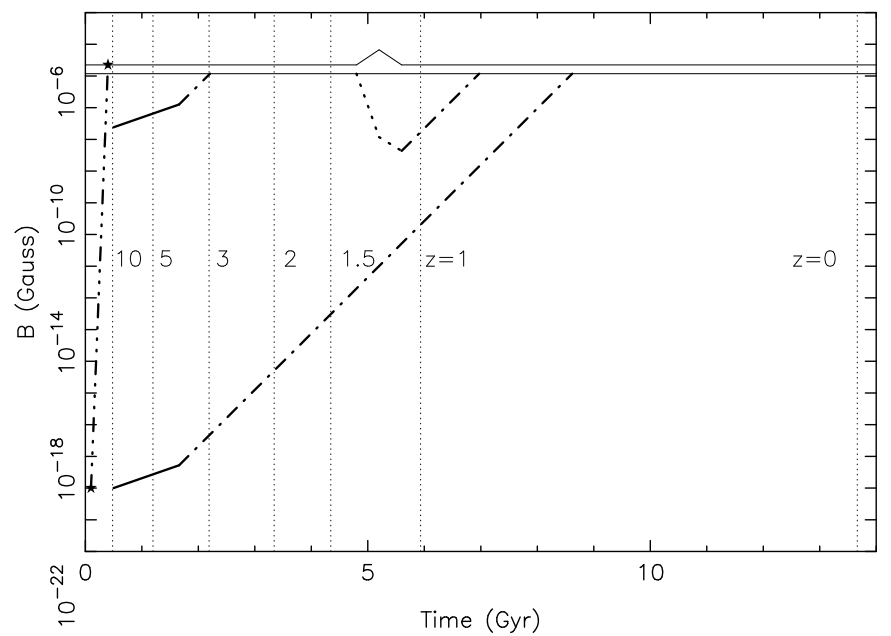

Fig. 1. Evolution of magnetic fields in MW-type disk galaxies: magnetic-field strength versus cosmic epoch. Evolution of the smallscale magnetic field generated by the turbulent dynamo (thick dasheddot-dot-dot line) and the large-scale magnetic field generated by the large-scale mean-field dynamo in quasi-spherical galaxies (thick solid line) or in thin-disk galaxies (thick dashed-dot-dashed). Dissipation of the field because of a major merger event is presented by a dotted line. The lower curve traces the evolution of regular magnetic fields generated by the pure large-scale dynamo mechanism (no amplification by the turbulent dynamo). The two horizontal thin solid lines represent the equipartition and equilibrium magnetic-field strengths (upper and lower lines, respectively). The vertical thin dotted lines indicate redshifts from 0 to 10 .

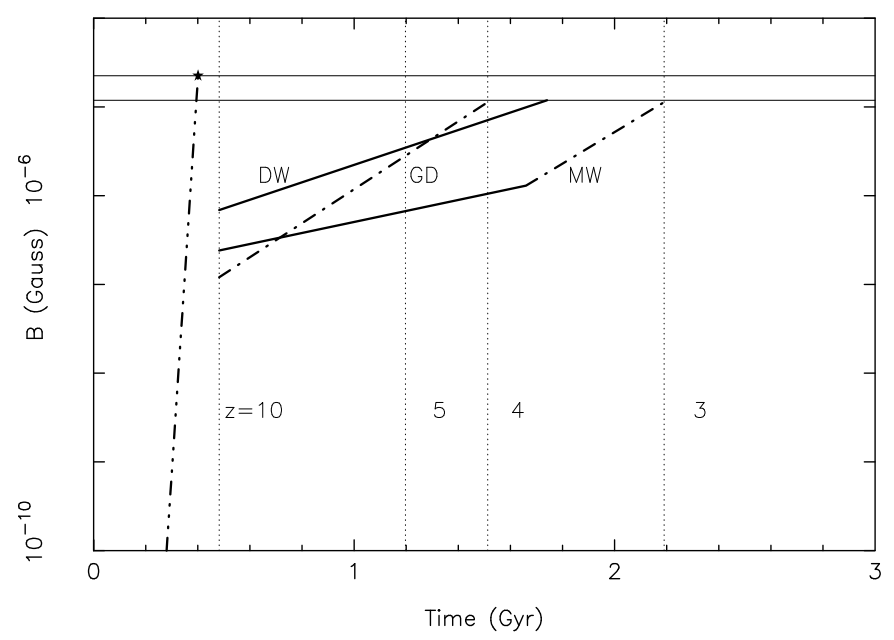

Fig. 2. Evolution in the magnetic-field strength of dwarf galaxies (DW), MW-type, and giant galaxies (GD). The meanings of different line types are the same as in Fig. 1.

disk-dominated galaxies and that their gas density and angular rotation velocity are unchanged during the evolution, i.e. $\rho=10^{-23} \mathrm{~g} \mathrm{~cm}^{-3}$ and $\Omega=20 \mathrm{~km} \mathrm{~s}^{-1} \mathrm{kpc}^{-1}$.

Halos. We assume that virial turbulence dominates during the epoch of merging and virialization of dark matter halos (Wise $\&$ Abel 2007). The typical turbulence velocity and the largest scale of turbulence, $20 \mathrm{~km} \mathrm{~s}^{-1}$ and $200 \mathrm{pc}$ in halos of masses $\lesssim 10^{8} M_{\odot}$ (John Wise, private communications) and are adopted for halos of masses $\lesssim 10^{12} M_{\odot}$.

Galaxies. For massive and MW-type disk galaxies $\left(\gtrsim 10^{10} M_{\odot}\right)$, we assume that (a) the gaseous and stellar

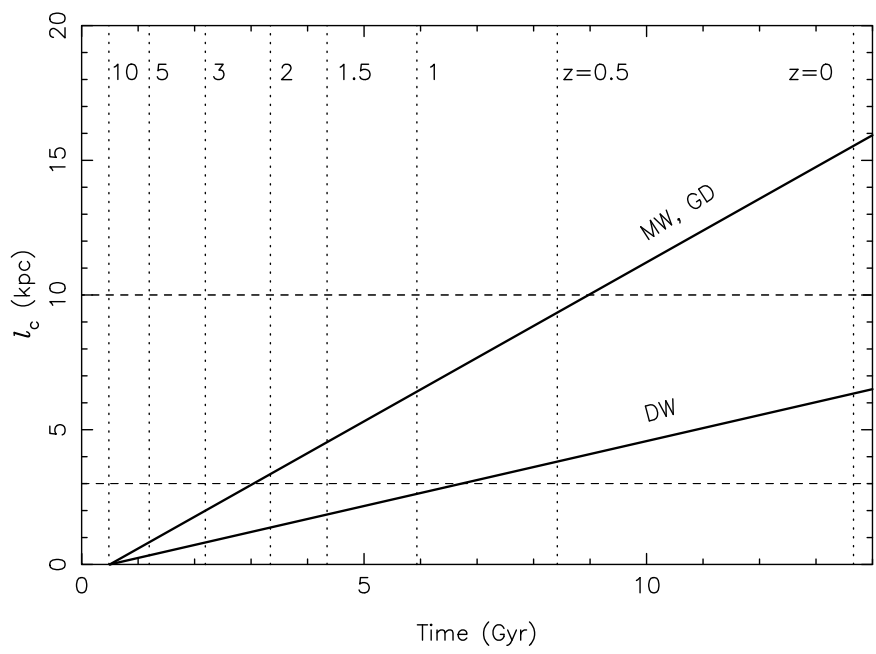

Fig. 3. Scale of ordering of regular magnetic fields with cosmological epoch. The evolution of the ordering magnetic fields is shown for dwarf galaxies (DW; bottom line) and disk galaxies (MW and GD; top line).

disks formed at $z_{\text {disk }} \approx 10$; (b) the turbulence scale and turbulence velocity were driven by $\mathrm{SN}$ explosions and had values close to those observed in present-day disk galaxies such as the MW, $l=100 \mathrm{pc}$ and $v=10 \mathrm{~km} \mathrm{~s}^{-1}$; (c) the scale height of the disk, $h=500 \mathrm{pc}$, remained unchanged as demonstrated by $N$-body simulations of late-type disk galaxies at $z<1$ (Brook et al. 2006), while its scale length in the plane decreased according to $R_{z}=R(1+z)^{-0.45}$ (Trujillo et al. 2006), where $R(z=0)=10 \mathrm{kpc}$. This is in reasonably good agreement with the observed disk thickness ratio of $h / R \sim 0.08$ at $z \sim 1$ (Reshetnikov et al. 2003) and $h / R \sim 0.15$ at higher redshifts (Elmegreen et al. 2005).

For typical low-mass puffy galaxies $\left(\leqslant 10^{10} M_{\odot}\right)$, we adopt $R=3 \mathrm{kpc}, h \approx R=3 \mathrm{kpc}$, and $\Omega=20 \mathrm{~km} \mathrm{~s}^{-1} \mathrm{kpc}^{-1}$. The ordering timescale of the field in these galaxies is calculated from Eq. (13) using $h \approx R$. We assume that the "quasi-spherical", mean-field dynamo amplified the regular magnetic field in puffy galaxies $(h / R>0.1)$, while the "disk" mean-field dynamo was effective in galaxies with a thin disk $(h / R<0.1)$.

\subsection{Isolated disk galaxies}

We consider the evolution of magnetic fields in giant disk galaxies (called GD hereafter; $R(z=0)=20 \mathrm{kpc}$ ), MW-type galaxies $(10 \mathrm{kpc})$, and dwarf galaxies (called DW hereafter; $R(z=$ $0)=3 \mathrm{kpc}$ ). We assume that the seeds of turbulent magnetic fields of strength $\approx 10^{-18} \mathrm{G}$ existed in the protogalaxies of present disk galaxies, at $z \approx 35$. Virial turbulence could amplify turbulent magnetic fields in merging dark-matter haloes via the small-scale dynamo from $10^{-18} \mathrm{G}$ seed field strength to reach the equipartition field strength of $2.2 \times 10^{-5} \mathrm{G}$ at $z \simeq 11(0.4 \mathrm{Gyr})$ within a short period of time, $\approx 3 \times 10^{8} \mathrm{yr}$ (Eq. (22); see Figs. 1 and 2). For simplicity, we consider that all types of galaxies reached the equipartition level of turbulent fields at the same epoch, $z \simeq 11$.

$M W$-like galaxies. A sketch of the evolution in the magnetic fields of isolated MW-type galaxies with $M_{\mathrm{MW}} \sim 10^{11} M_{\odot}$ is shown in Figs. 1 and 2 . At $z_{\text {disk }} \approx 10$, the disk was formed and evolved in isolation (no major merger) to the present time. The size of the galaxy was $R_{10}(z=10)=R(1+z)^{-0.45}=3.4 \mathrm{kpc}$ and $h / R_{10}=0.14>0.1$, and the "quasi-spherical" mean-field 
dynamo amplified the field until $z \approx 4$, at which time, $h / R$ became less then 0.1 . The "disk" mean-field dynamo then became significantly more important and amplified the regular largescale field within $\approx 1.5 \mathrm{Gyr}$, and reached its equilibrium state at $z \approx 3$ (Fig. 1). At this epoch, the regular field was ordered on a scale of a few kiloparsecs (Fig. 3) and finally reached a coherence scale similar to the size of the MW $(10 \mathrm{kpc})$ at $z \approx 0.4$.

The earliest regular magnetic fields of equipartition strength were generated in $t_{\text {d,global }} \approx 1.4 \mathrm{Gyr}$ after disk formation (Fig. 1), while the ordering of magnetic fields on a length scale similar to that of a MW-type galaxy was complete after $\approx 9$ Gyr (Fig. 3). Hence, present-day, MW-type galaxies are expected to host fully ordered regular fields.

This evolutionary scenario also corresponds to the evolution of a disk galaxy that experiences multiple minor mergers that do not destroy its disk. Multiple and even multiple frequent minor mergers may have caused local distortion of the disk, resulting in a high star-formation rate that shifted the start of large-scale dynamo action to a later epoch. This will be discussed in more detail in the next Section.

If amplification by the turbulent dynamo is not considered, the mean-field, large-scale dynamo would have to amplify a seed field of $10^{-18} \mathrm{G}$, and the time to reach the equilibrium strength of the regular magnetic field would be far longer $(\approx 9 \mathrm{Gyr}$, until a redshift of $z \approx 0.3$ ) (Fig. 1). However, this scenario is unrealistic because sufficient turbulence exists in young galaxies to drive the turbulent dynamo. Even without turbulence, another mechanism may enhance the seed field strength by a factor of $\gtrsim 10^{4}$ : the dissipative collapse into a disk (Lesch \& Chiba 1995) at $z \approx 10$. This would enable the equipartition level to be reached earlier, within $\approx 7.5 \mathrm{Gyr}$. In case of turbulence, this process is not required because the turbulent field is already strong at this redshift. However, if the collapse of the halo occurs at $z \gtrsim 10$, when the field amplitude remains smaller than the equipartition level (see Fig. 1), this mechanism may further accelerate the field amplification by the turbulent dynamo. In the following, we only consider amplification of the seed field by the turbulent dynamo.

Giant galaxies. Late-type disk galaxies of a disk scale length $\gtrsim 10 \mathrm{kpc}$ are rare as verified by analyses of $I$-band images (Courteau et al. 2007). In these giant disk galaxies $\left(M_{\mathrm{GD}} \sim\right.$ $\left.10^{12} M_{\odot}\right)$, the ratio $h / R_{10}(z=10)=0.5 / 6.8<0.1$ implies that the "disk" mean-field dynamo has already been switched on at $z \approx 10$ (Fig. 2), had amplified the regular magnetic field within only $\approx 1 \mathrm{Gyr}$, and reached the equilibrium state already at $z \approx 4$. However, as shown in Fig. 3, the regular magnetic fields can be ordered only on scales of $15 \mathrm{kpc}$ until the present time. Hence, the mean-field dynamo cannot generate coherent magnetic fields over the size of the disks of giant galaxies $(R \gtrsim 15 \mathrm{kpc})$.

Dwarf galaxies. We assume that, in low-mass halos, the turbulent magnetic fields evolve in the same way as in their massive counterparts. $N$-body/smoothed particle hydrodynamics simulations demonstrated that low-mass galaxies did not at first form thin disks because of the ionizing UV background radiation, which prevented cooling of the warm galactic gas (Kaufmann et al. 2007). They were more spheroidal, puffy systems $(h / R \approx$ 0.3 ) of rotational velocities $\approx 40 \mathrm{~km} \mathrm{~s}^{-1}$ and mass $M \approx 10^{10} M_{\odot}$. In thick galaxies, the "quasi-spherical" dynamo could generate and amplify the regular magnetic field to the equilibrium field strength within $\approx 1.5 \mathrm{Gyr}$ (at $z \approx 3.5$; Fig. 2 ). At $z \approx 2$, the UV background intensity decreases (Bianchi et al. 2001), resulting in the formation of a thin disk in a small galaxy that preserves the strength and ordering of the existing regular field. Although the ordering timescale of dwarf galaxies is longer (Fig. 3), fully coherent regular magnetic fields were generated at earlier epochs $(z \approx 1)$ because of the smaller sizes of dwarf galaxies.

Due to the hierarchical clustering, it is expected that more massive protogalaxies formed at later epochs. In this scenario, the first disks should have formed in MW-type galaxies (see Fig. 2) and, hence, the time to reach magnetic-field strengths of $\sim 10^{-6} \mathrm{G}$ in MW-type and giant galaxies would be comparable, while the ordering of the regular fields would have been completed earlier in MW-type galaxies. If dwarf galaxies formed earlier $(z \gtrsim 10)$, the regular field would have reached the equilibrium magnetic-field strength earlier and would have been ordered even earlier, at $z \approx 1$ (Fig. 3).

\subsection{Influence of star formation and mergers}

Star formation. The evolution of regular magnetic fields depends on the star-formation rate (SFR) and the disk parameters. Models of disk formation demonstrated that star formation is a fundamental parameter. It can be triggered in isolated galaxies by gravitational instability, in interacting galaxies by minor and major mergers, by tidal forces, leading to the compression of the gas, and by interactions of high velocity HI diffuse clouds (e.g. Kennicutt et al. 1987; Combes 2005). Star formation is more efficient at high redshifts because of the high merging rate and more gas at $z \simeq 1.3$ (Lefevre et al. 2000; Ryan et al. 2008), and in more massive disk galaxies (Governato et al. 2007).

Major mergers of gas-rich galaxies may trigger high SFR, causing high velocity turbulence of the ionized gas, which in turn can suppress the mean-field dynamo in the thick disk if the dynamo number is $\left|D_{\mathrm{d}}\right|<D_{\mathrm{c}} \approx 100-300$ and in the thin disk if $\left|D_{\mathrm{c}}\right|<D_{\mathrm{c}} \approx 7$ (see Sects. 2.3 and 2.1). The latter restriction places an upper limit on the turbulent velocity of the gas:

$v \lesssim 1.1 \Omega h=11 \mathrm{~km} \mathrm{~s}^{-1}$,

where $\Omega=20 \mathrm{~km} \mathrm{~s}^{-1} \mathrm{kpc}^{-1}$ and $h=0.5 \mathrm{kpc}$. The turbulent velocity (or characteristic velocity dispersion of the gas) and the SFR correlate positively for nearby galaxies (Dib et al. 2006). Using this relation and Eq. (26), one derives an upper limit of $S F R \lesssim 20$ (in units of $M_{\odot} \mathrm{yr}^{-1}$ ) up to which the action of the large-scale dynamo is possible. This indicates that the merging history of the galaxy had an important influence on the evolution of magnetic fields. In this scenario, regular fields were generated primarily at later epochs, $z<4$ (see Fig. 1).

Major mergers were rare but could alter the morphology of a spiral galaxy and destroy its regular magnetic field. After the merger, there were two possibilities, firstly, to form a disk galaxy with spheroidal or bulge component, or secondly, to form an elliptical galaxy without a disk. The SFR during the major merger $\left(\$ 2 \times 10^{9} \mathrm{yr}\right)$ was $\sim 50$ times higher than in the case of an isolated galaxy (di Matteo et al. 2007; Bournaud et al. 2007). This high SFR produced an increase in total magnetic-field strength as shown by the peak of the equipartition magnetic-field strength at about 5 Gyr in Fig. 1.

Disk galaxies, which survived after a gas-rich major merger, formed a thin-disk component immediately after the merger event and needed $\approx 1.5$ Gyr to amplify the regular magnetic field to the equilibrium level by the mean-field dynamo (Fig. 1) and $\approx 8 \mathrm{Gyr}$ to generate a coherent magnetic field on the length scale of the galaxy size (Fig. 3).

Minor mergers were more frequent and may also have altered the morphology (spiral into elliptical, spiral to spheroid), increased the size and thickness of the disk, and controlled the star formation rate (density, turbulence velocity). Simulations of 
the formation of thick disks by minor mergers of satellites with a thin disk showed that mergers with 10-20\% mass of the mass of the host galaxy resulted in the formation of thick disks. The disk was not destroyed but considerably heated up, tilted, and flared (Villalobos et al. 2008). The scale length increased slightly with respect to the original host disk, while the scale height became up to four times larger, depending on the inclination of the satellite's orbit. A larger disk height and radius increased both the dynamo timescale (Eq. (7)) and the ordering timescale (Eq. (13)), and shifted the formation of regular magnetic fields to later epochs.

Multiple minor mergers. Depending on the mass ratio of galaxies and the number of minor mergers, the disk could have been preserved, forming a spheroidal component, or destroyed, forming multiple spheroids or an elliptical galaxy (Bournaud et al. 2007). The disk-formation model presented by Stringer \& Benson (2007) illustrated that the resulting disk of an isolated galaxy and a galaxy formed by minor mergers have a very similar evolutionary behaviors in which the main governing parameter is the infalling gas supplied from the hot halo.

\section{Discussion}

\subsection{Observational tools}

The radio - far-infrared correlation provides a powerful tool for detecting star formation in distant galaxies with the help of ground-based radio observations. However, its validity depends critically on the magnetic-field strength coupled with the interstellar gas density and, hence the star-formation rate (Helou \& Bicay 1993; Lisenfeld et al. 1996; Niklas \& Beck 1997). In galaxies with recent starbursts, the radio continuum emission can be deficient with respect to the far-infrared emission (Roussel et al. 2003). Either the cosmic rays or the magnetic field or both are generated with some time delay with respect to the dustheating radiation field.

The total magnetic field can be measured by the observed total power radio emission, corrected for the thermal fraction of a galaxy. The regular magnetic field can be traced by polarized synchrotron emission and by Faraday rotation. While Faraday rotation is an unambiguous signature of coherent regular fields, polarized emission can also emerge from "anisotropic" fields, which are the result of compressing or shearing of isotropic turbulent fields. This process has been observed in several galaxies of the Virgo cluster (Vollmer et al. 2007; Wezgowiec et al. 2007).

\subsection{Comparison with observations of galaxies}

Regular magnetic fields, as traced by polarized synchrotron emission and Faraday rotation, were detected in the disks of nearby spiral galaxies, with spiral patterns and amplitudes of up to $15 \mu \mathrm{G}$, and were generally strongest in interarm regions (Krause 1990; Beck 2005). Mean-field dynamo theory, in spite of its simplifications, reproduces successfully the basic spiral pattern of magnetic fields observed in nearby spiral galaxies as well as in barred galaxies (Beck et al. 1996; Kulsrud 1999; Rüdiger \& Hollerbach 2004; Moss et al. 2007). The prominent example is M31, which hosts a coherent axisymmetric field within the emission "ring" at about $10 \mathrm{kpc}$ radius (Fletcher et al. 2004). However, regular fields with a coherence scale similar to the galaxy size are rare among nearby galaxies.

In contrast to the turbulent dynamo, the mean-field dynamo needs far more time to generate a regular field. Fields with a coherence length of about $1 \mathrm{kpc}$ can be expected after 1-2 Gyr, at $z \simeq 3-4$ (Fig. 3), but the generation time for regular fields with a coherence length of the galaxy size is several times longer and comparable to the galaxy age (Fig. 3). The ordering timescale for a $10 \mathrm{kpc}$ coherence scale such as that in M31 is about $10 \mathrm{Gyr}$ (Fig. 3). The coherent field of M31 is an indication that this galaxy has not experienced from a major merger during the past 10 Gyr.

Most other nearby galaxies observed so far have more complicated field patterns than in M31 (Beck 2005). The long timescale required for field ordering (Fig. 3) is consistent with these observations: most galaxies probably did not yet reach full field coherence, either due to their size or due to major merging events. The lifetime of galaxies larger than $15 \mathrm{kpc}$ is insufficient to generate a fully coherent field. If a major merger in a MWtype galaxy occurred less than about 5 Gyr ago, the recovery of the fully coherent regular field is not yet completed.

The results of this paper suggest a correlation between coherence scale and galaxy size. The only direct indication of a regular field in a distant galaxy was derived from Faraday rotation in an intervening galaxy at $z=0.395$ measured against a background quasar (Kronberg et al. 1992). Since the pattern exhibits reversals and hence is incoherent on the galaxy scale, this result is consistent with our results. Indirect evidence of regular fields in foreground spiral systems beyond $z \simeq 1$ has been provided by Faraday rotation probes of distant radio sources (Kronberg et al. 2008; Bernet et al. 2008). They found that the scatter in the intrinsic RMs of spirals is $\sim 20-50 \mathrm{rad} \mathrm{m}^{-2}$, which implies a coherence of the field on scales of about half the galaxy size (in the case of no field reversals). This coherence scale is consistent with the ordering scale of magnetic fields derived in this work (see Fig. 3) for MW-type galaxies in the redshift range $z \approx 1-2$.

In the LMC, another axisymmetric field was detected, which was found to have properties conflicting with dynamo theory (Gaensler et al. 2005). Since the LMC has a disk, although this rotates slowly, the mean-field dynamo can indeed operate and create a global coherent field on a scale of $4 \mathrm{kpc}$ (radius of the LMC) within about 10 Gyr (Fig. 3). Hence, dwarf galaxies are prime candidates for hosting fully coherent regular fields. On the other hand, dwarf galaxies are affected more significantly than larger galaxies by interactions. Simulations indicate that the LMC may have passed through the plane of the Milky Way more than 2 Gyr ago, or that it is on its way to a first passage through the MW (Kallivayalil et al. 2006; Piatek et al. 2008, and references therein). Since the regular field would be severely distorted during each passage, the observation of a regular field supports the idea of no passages in the past.

M 82 is the prototypical and most nearby starburst galaxy, excited by a tidal interaction with M 81. Its total magnetic field is strong, about $50 \mu \mathrm{G}$ (Klein et al. 1988), and probably in equipartition with the other components of the wind outflow. The radio emission from the galaxy disk appears to be almost completely depolarized (Reuter et al. 1994). The radio halo is highly polarized but Faraday rotation is small, indicating anisotropic turbulent fields that are sheared and compressed in the rapidly moving gas outflow. This confirms our model of a strong and dominant turbulent field in starburst galaxies (Fig. 1). Observations of higher frequency, however, also detected polarized emission in the disk (Wielebinski 2006), indicating that some regular disk field may have survived the tidal interaction, which might not have been the case after a galaxy merger. To search for regular fields in the disks and halos of starburst galaxies, new observations of higher resolution are required.

From Zeeman splitting in the HI absorption line against, a background quasar (Wolfe et al. 2008) a $84 \pm 9 \mu \mathrm{G}$ field with a 
coherence length of $\geq 200$ pc was discovered in a low-metallicity galaxy at $z=0.692$. A turbulent dynamo can generate such a strong turbulent field in a starburst galaxy triggered by a major merger (see Fig. 1). However, this field cannot produce a Zeeman signal because of line-of-sight integration through the whole galaxy, unless the observed field is located in a single dense gas cloud or filament. Alternatively, the observed field could originate in a shear or compression layer between interacting galaxies, although verification of this possiblity is beyond the scope of this paper.

\subsection{Detection of synchrotron emission from distant galaxies with SKA}

One major objective of SKA observations will be the detection of radio continuum emission from distant galaxies to study the star-formation history in the early Universe (van der Hulst et al. 2004). The tight radio - far-infrared correlation in galaxies implies that radio synchrotron emission is an excellent tracer of star formation in galaxies (Condon et al. 1991), at least to distances of $z \simeq 3$ (Seymour et al. 2008). However, its application to even higher redshifts depends crucially on the existence of magnetic fields at the equipartition level with turbulent gas motions.

The timescale for field generation in young galaxies constrains the largest distance to which SKA can detect star-forming galaxies in synchrotron emission. We have shown in this paper that, even in the case of very weak seed fields of $10^{-18} \mathrm{G}$, the turbulent small-scale dynamo, with the help of virial turbulence, can amplify turbulent fields to the level of equipartition with turbulent energy density within $\simeq 3 \times 10^{8}$ years; strong fields should therefore exist in all star-forming galaxies at $z \simeq 10$ (Fig. 1) and the radio - far-infrared correlation should be valid for $z \lesssim 10$. Hence, radio continuum emission can be used as a tracer of star formation in the early Universe.

Star-formation rate and total magnetic-field strength are related nonlinearly (Niklas \& Beck 1997; Chyży et al. 2007; Krause 2008), so that young starburst galaxies should have much stronger fields and a higher radio - far-infrared ratio than normal galaxies. The SKA and its pathfinder telescopes will investigate this relation in more detail.

The SKA will detect radio continuum emission from spiral galaxies similar to the MW to $z \simeq 3$ and to even larger distances for more massive and starburst galaxies. The prediction from dynamo models presented in this paper can be tested with the help of polarization and Faraday rotation data from the SKA radio continuum and RM surveys (Gaensler et al. 2004). Systematic observations of galaxies at $z=1-5$ are expected to envisage the early stages of galactic magnetic-field evolution.

The first regular magnetic fields of contemporary disk galaxies were formed at $z \approx 4$, when the $h / R$ ratio was higher than today. Therefore, their field patterns may have been substantially different from those of local galaxies (Moss \& Sokoloff 2008), and their coherence scales were still smaller than their sizes. Our model predicts that dwarf and giant galaxies have generate regular fields of several $\mu \mathrm{G}$ strength by $z \approx 4$, while MW-type galaxies should not host these fields before $z \approx 3$. An important implication is that polarized radio disks and Faraday rotation are expected to exist in all galaxies at high redshifts $(z \lesssim 3)$. Even if these galaxies cannot be resolved by the SKA beam, the integrated radio emission should be significantly polarized for moderate and high inclinations (Stil et al. 2008). A statistical analysis of a significant sample of galaxies observed by the SKA surveys would be able to test the validity of the predictions of this paper. The detailed field pattern can be measured with the help of a dense RM grid of polarized background sources (Stepanov et al. 2008).

Major merging events destroy the field regularity, while the total equipartition field strength is increased over a certain period (see Fig. 1 and Sect. 6.4). Furthermore, violent forces during the merging process can compress the turbulent field and lead to strong polarized emission. Hence, the polarized intensity increases, although the large-scale coherent field will be weaker than before. Faraday rotation measurements are required to distinguish coherent from anisotropic fields.

Some fraction of galaxies will be observed in a post-merger or starburst phase when the regular field has been temporarily destroyed and has not yet recovered (Fig. 1). A correlation between Faraday rotation and signatures of a recent merger is expected and should be investigated, whereas the total and polarized emission should be higher due to enhanced turbulence and a fast outflow. Our estimates constrain the time since the peak of the starburst and will be useful for observing distant, starburst galaxies.

Outflows from starburst galaxies contribute to the magnetization of the intergalactic medium (IGM) (Kronberg et al. 1999). Estimating the importance of this effect will require reliable statistical data about starburst frequency, e.g. from radio observations with SKA.

\section{Conclusions}

Studying the evolution in magnetic fields of galaxies is important for interpreting future radio synchrotron observations with the planned Square Kilometre Array (SKA). We have used the dynamo theory to derive the timescales of amplification and ordering of magnetic fields in disk and quasi-spherical galaxies. This has provided a useful tool in developing a simple evolutionary model of regular magnetic fields, coupled with models describing the formation and evolution of galaxies. In the epoch of dark matter halo formation, seed magnetic fields of $\sim 10^{-18} \mathrm{G}$ strength were generated in protogalaxies by the Biermann battery. Turbulence in the protogalactic halo generated by thermal virialization could have driven the turbulent (small-scale) dynamo and amplify the seed field to the equipartition level of $\approx 20 \mu \mathrm{G}$ within a few $10^{8} \mathrm{yr}$. In the epoch of disk formation, the turbulent field served as a seed for the mean-field (large-scale) dynamo developed in the disk.

- We defined three characteristic timescales for the evolution of galactic magnetic fields: one for the amplification of the seed field, a second for the amplification of the large-scale regular field, and a third for the field ordering on the galactic scale.

- Galaxies similar to the Milky Way formed their disk at $z \approx$ 10. Regular fields of equipartition ( $\operatorname{several} \mu \mathrm{G})$ strength and a few kpc coherence length were generated within 2 Gyr (until $z \approx 3$ ), but field ordering up to the coherence scale of the galaxy size took another 6 Gyr (until $z \approx 0.5$ ).

- Giant galaxies had already formed their disk at $z \gtrsim 10$, allowing more efficient dynamo generation of equipartition regular fields (with a coherence length of about $1 \mathrm{kpc}$ ) until $z \approx 4$. However, the age of the Universe is too young for fully coherent fields to have already developed in giant galaxies larger than about $15 \mathrm{kpc}$.

- Dwarf galaxies formed even earlier and should have hosted fully coherent fields at $z \approx 1$.

- Major mergers excited starbursts with enhanced turbulence, which in turn amplified the turbulent field, whereas the 
regular field was disrupted and required several Gyr to recover. Measurement of regular fields can serve as a clock for measuring the time since the last starburst event.

- Starbursts due to major mergers enhance the turbulent field strength by a factor of a few and drive a fast wind outflow, which magnetizes the intergalactic medium. Observations of the radio emission from distant starburst galaxies can provide an estimate of the total magnetic-field strength in the IGM.

This evolutionary scenario can be tested by measurements of polarized synchrotron emission and Faraday rotation with the SKA. We predict an anticorrelation at fixed redshift between galaxy size and the ratio between ordering scale and the galaxy size. Weak regular fields (small Faraday rotation) in galaxies at $z \lesssim 3$, possibly associated with strong anisotropic fields (strong polarized emission), would be signatures of major mergers. Undisturbed dwarf galaxies should host fully coherent fields, giving rise to strong Faraday rotation signals.

Acknowledgements. This work is supported by the European Community Framework Programme 6, Square Kilometre Array Design Study (SKADS) and the DFG-RFBR project under grant 08-02-92881. We acknowledge valuable discussions with Lucio Mayer, Klaus Dolag and John Wise who provided also simulations of the density and power spectrum of halos. We thank Philip Kronberg for careful reading the manuscript and valuable discussions.

\section{References}

Batchelor, G. K. 1950, Proc. R. Soc. Lond., A201, 405

Baugh, C. M., Cole, S., \& Frenk, C. S. 1996, MNRAS, 283, 1361

Beck, R. 2005, in Cosmic Magnetic Fields, ed. R. Wielebinski, \& R. Beck (Berlin: Springer), 43

Beck, R., Poezd, A. D., Shukurov, A., \& Sokoloff, D. D. 1994, A\&A, 289, 94

Beck, R., Brandenburg, A., Moss, D., Shukurov, A., \& Sokoloff, D. 1996, ARA\&A, 34, 155

Beck, R., Shukurov, A., Sokoloff, D., \& Wielebinski, R. 2003, A\&A, 411, 99

Belvedére, G., Lanza, A., \& Sokoloff, D. 1998, Sol. Phys., 183, 435

Bernet, M. L., Miniati, F., Lilly, S. J., Kronberg, P. P., \& Dessauges-Zavadsky, M. 2008, Nature, 454, 302

Bianchi, S., Cristiani, S., \& Kim, T.-S. 2001, A\&A, 376, 1

Binney, J., \& Tremaine, S. 1987, Galactic dynamics (Princeton: University Press)

Birnboim, Y., \& Dekel, A. 2003, MNRAS, 345, 349

Bournaud, F., Jog, C. J., \& Combes, F. 2007, A\&A, 476, 1179

Brook, C. B., Kawata, D., Gibson, B. K., \& Freeman, K. C. 2004, ApJ, 612, 894

Chyży, K. T., Bomans, D. J., Krause, M., et al. 2007, A\&A, 462, 933

Combes, F. 2005, in The Evolution of Starbursts, Amer. Inst. Phys. Conf. Ser. 783,43

Condon, J. J., Anderson, M. L., \& Helou, G. 1991, ApJ, 376, 95

Courteau, S., Dutton, A. A., van den Bosch, F. C., et al. 2007, ApJ, 671, 203

Davies, G., \& Widrow, L. M. 2000, ApJ, 540, 755

de Avillez, M. A., \& Breitschwerdt, D. 2005, A\&A, 436, 585

Dekel, A., \& Birnboim, Y. 2006, MNRAS, 368, 2

di Matteo, P., Combes, F., Melchior, A.-L., \& Semelin, B. 2007, A\&A, 468, 61

Dib, S., Bell, E., \& Burkert, A. 2006, ApJ, 638, 797

Elmegreen, B. G., Elmegreen, D. M., Vollbach, D. R., Foster, E. R., \& Ferguson, T. E. 2005, ApJ, 634, 101

Elmegreen, D. M., Elmegreen, B. G., Ravindranath, S., \& Coe, D. A. 2007, ApJ, 658,763

Fletcher, A., Berkhuijsen, E. M., Beck, R., \& Shukurov, A. 2004, A\&A, 414, 53

Frick, P., Stepanov, R., \& Sokoloff, D. 2006, Phys. Rev. E, 74, 066310

Gaensler, B. M., Beck, R., \& Feretti, L. 2004, New Astr. Rev., 48, 1003

Gaensler, B. M., Haverkorn, M., Staveley-Smith, L., et al. 2005, Science, 307, 1610

Genzel, R., Tacconi, L. J., Eisenhauer, F., et al. 2006, Nature, 442, 786

Governato, F., Willman, B., Mayer, L., et al. 2007, MNRAS, 374, 1479

Greif, T. H., Johnson, J. L., Klessen, R. S., \& Bromm, V. 2008 [arXiv:0803.2237]

Gressel, O., Elstner, D., Ziegler, U., \& Rüdiger, G. 2008, A\&A, 486, L35

Hanasz, M., Lesch, H., Otmianowska-Mazur, K., \& Kowal, G. 2005, in The Magnetized Plasma in Galaxy Evolution, ed. K. T. Chyzy, K. Otmianowska-

Mazur, M. Soida, \& R.-J. Dettmar, Jagiellonian University, Krakow, 162

Harrison, E. R. 1970, MNRAS, 147, 279
Helou, G., \& Bicay, M. D. 1993, ApJ, 415, 93

Iskakov, A. B., Schekochihin, A. A., Cowley, S. C., McWilliams, J. C., Proctor,

M. R. E. 2007, Phys. Rev. Lett., 98, 501

Ivison, R. J., Smail, I., Dunlop, J. S., et al. 2005, MNRAS, 364, 1025

Julian, W. H., \& Toomre, A. 1966, ApJ, 146, 810

Kallivayalil, N., van der Marel, R. P., \& Alcock, C. 2006, ApJ, 652, 1213

Kauffmann, G., White, S. D. M., \& Guiderdoni, B. 1993, MNRAS, 264, 201

Kaufmann, T., Wheeler, C., \& Bullock, J. S. 2007, MNRAS, 382, 1187

Kazantsev, A. P. 1967, JETP, 53, 1806 [Sov. Phys. JETP, 26, 1031, 1968]

Kennicutt, R. C., Jr., Roettiger, K. A., Keel, W. C., van der Hulst, J. M., \& Hummel, E. 1987, AJ, 93, 1011

Kitchatinov, L. L., \& Rüdiger, G. 2004, A\&A, 424, 565

Kleeorin, N., Moss, D., Rogachevskii, I., \& Sokoloff, D. 2002, A\&A, 387, 453

Klein, U., Wielebinski, R., \& Morsi, H. W. 1988, A\&A, 190, 41

Kolmogorov, A. N., Petrovsky, I. G., \& Piskunov, N. S. 1937, Bull. Moscow Univ., A1, 1

Korpi, M. J., Brandenburg, A., Shukurov, A., Tuominen, I., \& Nordlund, Å 1999, ApJ, 514, L99

Kowal, G., Otmianowska-Mazur, K., \& Hanasz, M. 2006, A\&A, 445, 915

Krause, M. 1990, in Galactic and Intergalactic Magnetic Fields, ed. R. Beck, R. Wielebinski, P. P. Kronberg, IAU Symp., 140, 187

Krause, M. 2008 [arXiv:0806.2060]

Kronberg, P. P. 1994, Rep. Progr. Phys., 57, 325

Kronberg, P. P., Perry, J. J., \& Zukowski, E. L. H. 1992, ApJ, 397, 528

Kronberg, P. P., Lesch, H., \& Hopp, U. 1999, ApJ, 511, 56

Kronberg, P. P., Bernet, M. L., Miniati, F., et al. 2008, ApJ, 676, 70

Kulsrud, R. M. 1999, ARA\&A, 37, 37

Landau, L. D., \& Lifshitz, E. M. 1959, Fluid Mechanics (Addison-Wesley, Reading, Mass)

Labbé, I., Rudnick, G., Franx, M., et al. 2003, ApJ, 591, L95

Le Fèvre, O., Abraham, R., Lilly, S. J., et al. 2000, MNRAS, 311, 565,

Lesch, H., \& Chiba, M. 1995, A\&A, 297, 305

Lisenfeld, U., Völk, H. J., \& Xu, C. 1996, A\&A, 314, 745

Mayer, L., Governato, F., \& Kaufmann, T. 2008 [arXiv:0801. 3845]

Medvedev, M. V., Silva, L. O., Fiore, M., Fonseca, R. A., \& Mori, W. B. 2004,

J. Korean Astron. Soc., 37, 533

Meneguzzi, M., Frisch, U., Pouquet, A. 1981, Phys. Rev. Lett., 47, 1060

Mishustin, I. N., \& Ruzmaikin, A. A. 1971, Sov. Phys. JETP, 61, 441

Moss, D., \& Sokoloff, D. 2008, A\&A, 487, 197

Moss, D., Snodin, A. P., Englmaier, P., Shukurov, A., Beck, R., \& Sokoloff, D. 2007, A\&A, 465, 157

Moss, D., Shukurov, A., \& Sokoloff, D. 2008, Geophys. Astrophys. Fluid Dyn., 89,285

Niklas, S., \& Beck, R. 1997, A\&A, 320, 54

Omukai, K., \& Palla, F. 2003, ApJ, 589, 677

Piatek, S., Pryor, C., \& Olszewski, E. W. 2008, AJ, 135, 1024

Press, W. H., \& Schechter, P. 1974, ApJ, 187, 425

Pudritz, R. E., \& Silk, J. 1989, ApJ, 342, 650

Rees, M. J. 2006, Astron. Nachr., 327, 395

Rees, M. J., \& Ostriker, J. P. 1977, MNRAS, 179, 541

Reshetnikov, V. P., Dettmar, R.-J., \& Combes, F. 2003, A\&A, 399, 879

Reuter, H.-P., Klein, U., Lesch, H., Wielebinski, R., \& Kronberg, P. P. 1994, A\&A, 282, 724

Roussel, H., Helou, G., Beck, R., et al. 2003, ApJ, 593, 733

Rüdiger, G., \& Hollerbach, R. 2004, The Magnetized Universe. Geophysical and Astrophysical Dynamo Theory (Wiley-VCH, ESO)

Ruzmaikin, A., Shukurov, A., \& Sokoloff, D. 1988, Magnetic Fields of Galaxies (Dordrecht: Kluwer)

Ryan, R. E., Jr., Cohen, S. H., Windhorst, R. A., \& Silk, J. 2008, ApJ, 678, 751

Schlickeiser, R., \& Shukla, P. K. 2003, ApJ, 599, L57

Semikoz, V. B., \& Sokoloff, D. D. 2005, Int. J. Mod. Phys. D, 14, 1839

Seymour, N., Dwelly, T., Moss, D., et al. 2008, MNRAS, 386, 1695

Shukurov, A. 2004, in Mathematical aspects of Natural Dynamos

[arXiv:astro-ph/0411739]

Shukurov, A. 2005, in Cosmic Magnetic Fields, ed. R. Wielebinski, \& R. Beck (Berlin: Springer), 113

Sokoloff, D. 1995, Magnetohydrodynamics, 31, 43

Sokoloff, D. 2002, Astron. Rep., 96, 871

Sokoloff, D., \& Shukurov, A. 1990, Nature, 347, 51

Sokoloff, D. D., Nefedov S. N., Ermash A. A., \& Lamzin, S. A. 2008, [arXiv: 0806.0746]

Springel, V., \& Hernquist, L. 2005, ApJ, 622, L9

Stepanov, R., Arshakian, T. G., Beck, R., Frick, P., \& Krause, M. 2008, A\&A, 480, 45

Stil, J. M., Krause, M., Beck, R., \& Taylor, A. R. 2008 [arXiv: 0810.2303]

Stringer, M. J., \& Benson, A. J. 2007, MNRAS, 382, 641

Subramanian, K. 1998, MNRAS, 294, 718 
Takahashi, K., Ichiki, K., Ohno, H., Hanayama, H., \& Sugiyama, N. 2006, Astron. Nachr., 327, 410

Takizawa, M. 2005, ApJ, 629, 791

Tegmark, M., Silk, J., Rees, M. J., Blanchard, A., Abel, T., \& Palla, F. 1997, ApJ, 474, 1

Tobias, S. M., \& Cattaneo, F. 2008, Phys. Rev. Lett., 101, 125003

Trujillo, I., Förster S., Natascha M., et al. 2006, ApJ, 650, 18

van der Hulst, J. M., Sadler, E. M., Jackson, C. A., et al. 2004, New Astr. Rev., 48,1221

Villalobos, Á., \& Helmi, A. 2008 [arXiv: 0803. 2323]

Vollmer, B., Soida, M., Beck, R., et al. 2007, A\&A, 464, L37

Wang, P., \& Abel, T. 2007 [arXiv:0712.0872]
Weibel, E. S. 1959, Phys. Rev. Lett. 2, 83

Wielebinski, R. 2006, Astron. Nachr. 327, 510

Wezgowiec, M., Urbanik, M., Vollmer, B., et al. 2007, A\&A, 471, 93

Wise, J. H., \& Abel, T. 2007, ApJ, 665, 899

Wise, J. H., Matthew, J., Turk, M. J., \& Abel, T. 2007 [arXiv : 0710. 1678]

Wolfe, A. M., Jorgenson, R. A., Robishaw, T., Heiles, C., \& Prochaska, J. X. 2008, Nature, 455, 638

Xu, H., O'Shea, B. W., Collins, D. C., Norman, M. L., Li, H., \& Li, S. 2008 [arXiv: 0807.2647]

Zeldovich, Ya. B., Ruzmaikin, A. A., \& Sokoloff, D. D. 1990, The Almighty Chance (Singapore: World Science) 